\title{
Association between functional neurometry test results and blood biomarkers indicative of anaemia, subclinical inflammation, and endothelial dysfunction
}

\begin{abstract}
Introduction: Functional neurometry-FN assesses the functioning of the autonomic nervous system.

Objective: The association between FN test results and the biomarkers of anaemia, subclinical inflammation-SI and endothelial dysfunction-ED was evaluated.

Method: 412 male patients aged between 18-50 years were evaluated by FN and Laboratory Testing. Groups: control (CONT, $\mathrm{n}=194)$ and dysfunctional (DYSF, $\mathrm{n}=218)$. FN categories: Anxiety Control-(AC) $\geq 75 \%$, Baroreflex Index-(BRI) $\geq 90 \%$, Hemodynamic(HD) $\leq 10 \%$ and Physiological response-(PR) $=31.5^{\circ}-32.5^{\circ}$, expressed in Median (Minimum-Maximum). Biomarkers: red blood cells-RBC, haemoglobin, haematocrit, mean corpuscular volume-MCV; mean corpuscular haemoglobin-MCH; mean corpuscular haemoglobin concentration-MCHC; red cell distribution width-RDW; total leukocytes-TL, ferritin, transferrin saturation-TS, B12, homocysteine, C-reactive protein-CRP; fibrinogen; 25-hydroxy-vitamin-D3; apolipoprotein A1-Apo-A1; apolipoprotein B-Apo-B; glutamic oxalacetic transaminase-GOT; glutamic-pyruvic transaminase-GPT; Gamma GT; 8am cortisol; IgE; total cholesterol-TC; triglycerides-TG, and; high-density lipoprotein-HDL and low-density lipoprotein-LDL, expressed in frequency-[percentage] and analysed by the Chi-square test with $\mathrm{p} \leq 0.05^{*}$.
\end{abstract}

Results: $\quad$ DYSF $\quad$ presented: $\quad \mathrm{AC}=24.91 /(10.11-69.17), \quad \mathrm{BRI}=86.54 /(67.33-87.82)$, $\mathrm{HD}=17.89 /(15.74-24.41), \mathrm{PR}=24.98 /(22.60-28.60)$ and higher frequencies of patients with dysfunctional biomarkers: RBC 21/218-[9.63\%]; haemoglobins $=94 / 218-[43.12 \%$ ]; haematocrits $=52 / 218-[23.85 \%] ; \quad \mathrm{MCV}=114 / 218-[52.29 \%] ; \quad \mathrm{RDW}=52 / 218-[23.85 \%]$; ferritin=42/218-[19.27\%]; B12=157/218-[72.02\%]; homocysteine $=135 / 218-[61.93 \%]$; GPT $=53 / 218-[24.31 \%] ; \quad$ Gamma-GT=94/218-[43.12\%]; $\quad$ cortisol=114/218-[52.29\%]; $\operatorname{IgE}=53 / 218-[24.31 \%] ; \quad \mathrm{TL}=177 / 218-[81.19 \%] ; \quad \mathrm{CRP}=85 / 218-[38.99 \%] ;$ fibrinogen=74/218-[33.94\%]; $\quad 25$-hydroxy-vitamin-D3=138/218-[63.30\%]; $\mathrm{TC}=125 / 218-[57.34 \%] ; \quad \mathrm{TG}=63 / 218-[28.90 \%] ; \quad \mathrm{HDL}=35 / 218-[16.06 \%] ; \quad$ LDL $=65 / 218$ [29.82\%]; $\quad$ TL/HDL $(>3.3)=138 / 218-[63.30 \%], \quad$ TG/HDL $(>2.5)=138 / 218-[63.30 \%]$, LDL/HDL $(>2.3)=42 / 218-[19.27 \%] \quad$ and Apo-B/Apo-A1 $(>0.69)=95 / 218-[63,30 \%]$ ratios, compared to controls: $\mathrm{AC}=75.88 /(72.05-85.00), \quad \mathrm{BRI}=92.80 /(89.00-95.98)$, $\mathrm{HD}=10.00 /(09.00-10.00), \quad \mathrm{PR}=32.25 /(29.00-33.73), \quad$ haemoglobins $=13 / 194-[6.70 \%]$, haematocrits $=5 / 194-[2.58 \%], \quad \mathrm{MCV}=14 / 194-[7.22 \%], \quad \mathrm{RDW}=2 / 194-[1.03 \%]$; ferritin=5/194-[2.58\%];B12=12/194-[6.19\%]; homocysteine $=4 / 194-[2.06 \%]$; GPT =14/194[7.22\%]; Gamma-GT=17/194-[8.76\%]; cortisol=21/194-[10.82\%]; $\operatorname{IgE}=4 / 194-[2.06 \%]$; $\mathrm{TL}=21 / 194-[10.82 \%] ; \quad \mathrm{CRP}=6 / 194-[3.09 \%] ; \quad$ fibrinogen $=4 / 194-[2.06 \%] ; \quad 25$-hydroxyvitamin-D3=9/194-[4.64\%)]; TC=22/194-[11.34\%]; TG=21/194-[28.90\%]; HDL=10/194[5.15\%]; LDL=14/194-[7.22\%], TC/HDL=14/194-[7.22\%], TG/HDL=6/194-[3.09\%], $\mathrm{LDL} / \mathrm{HDL}=5 / 194-[2.58 \%]$ and Apo-B/Apo-A1 $=22 / 194-[11.34 \%]$.

Conclusion: This study found a significant association between FN test results and biomarkers indicative of anaemia, SI and ED.

Keywords: functional neurometry, biomarkers, anaemia, subclinical inflammation, endothelial dysfunction

\author{
Volume I4 Issue 5 - 202I
}

\author{
Renata de Melo Guerra Ribas,' Valdenilson \\ Ribeiro Ribas,' Diélita Carla Lopes de \\ Oliveira,' Marcelo Tavares Viana,' Joyce \\ Gomes de Moraes,' Hugo André de Lima \\ Martins,' Carlos Frederico de Oliveira \\ Alves,' Nery Adamy Neto, Murilo Tolêdo \\ Calafange, ${ }^{2}$ Clenes de Oliveira Mendes \\ Calafange, ${ }^{2}$ Ana Elisa Toscano, ${ }^{2}$ Raul Manhães \\ de Castro ${ }^{2}$ \\ 'Brain Institute of Pernambuco (ICerPE), Brazil \\ ${ }^{2}$ Postgraduate Program in Neuropsychiatry and Behavioral \\ Sciences (Posneuro), Brazil
}

\begin{abstract}
Correspondence: Valdenilson Ribeiro Ribas, Senador Sérgio Guerra, 220,Apt. 132, Piedade - Jaboatão dos Guararapes, Tel +5581 99245-603I, Email ribas.professor@gmail.com
\end{abstract}

Received: September 27, 2021 | Published: October 25, 2021

\section{Introduction}

The Functional Neurometry of the author Nelson Alves Pereira Júnior is a methodology that enables an intercommunication between Biofeedback resources already studied and scientifically demonstrated, such as: sensor that captures heart rate variability (HRV), galvanic skin response, cardiac coherence (synchrony between the frequencies of the respiratory cycle, heart rate and blood pressure), peripheral temperature sensors, respiratory rate and heart rate meter. ${ }^{1}$
The author organized his Functional Neurometry method in an assessment established in 3 (three) positions (dorsal position; standing up and standing) and named the categories of this initial investigative protocol: Anxiety control; baroreflex index; Hemodynamic; Physiological response and brain neurometry. ${ }^{2}$

In the first category, entitled anxiety control, the non-cognitive response (not voluntarily controlled in a rational way) is measured, that is, the ANS response. ${ }^{3}$ The author uses galvanic skin response 
sensors (dermal conductance). ${ }^{4}$ These sensors apply a very small and imperceptible electrical voltage to the skin, specifically, on the volar surface of the index and middle fingers, where there are numerous sweat glands, allowing you to measure the impedance of the electrical current in microohm units. ${ }^{2}$

Another relevant category of this initial psychophysiological assessment concerns the functioning of baroceptors. ${ }^{5}$ The more the baroceptors are activated, the more functional oxygen is transported to the mitochondria, ${ }^{1}$ especially in the places where the mitochondria are more agglomerated, which are: brain, muscles and ganglia. ${ }^{6}$ However, it is important to clarify that functional oxygen is not oxygen saturation.

Oxygen saturation measures the amount of oxygen-bound haemoglobin compared to the amount of unbound haemoglobin. Hypoxemia can lead to many acute adverse effects on individual organ systems. Having oxygen in the blood does not guarantee that this oxygen is arriving in sufficient quantity in the mitochondria of the brain and all the muscles, that is, there may be a difficulty in transporting oxygen due to anaemia, ${ }^{7}$ subclinical inflammation ${ }^{8}$ or atherosclerosis. ${ }^{9}$

In the case of the brain, this organ still faces the difficulty of gravity in receiving oxygen transported by red blood cells that need to reach an equilibrium in normocytosis, because, for example, if there is a megaloblastic anaemia, the red cell will be so large that it may have difficulties in transporting this oxygen passing through capillaries.

Baroreflex pulses can be counted in Functional Neurometry in a statistical percentage called the baroreflex index by the author and this calculation only provides assurance that functional oxygen reached the vasomotor centres in sufficient quantities, when it counts $90 \%$ or more. ${ }^{2}$ This functioning with active and constant movement of these mechanoreceptors also seems to indicate that there is a good flow in the blood displacement also called hemodynamic at the level of blood microcirculation.

In the process of investigating the functioning of oxygen transport, the category entitled physiological response stands out, which also attests to the functionality of the sympathetic and parasympathetic systems made possible through the thermoregulation sensor located in the annular proximal phalanx. ${ }^{10}$

During the care of several volunteer patients at a clinic of the Instituto do Cérebro de Pernambuco (ICerPE), in the city of Recife/ $\mathrm{PE}$, a possible association was observed between the findings of the results of the Functional Neurometry tests, anaemia, subclinical inflammation and/ or endothelial dysfunction. Thus, this study aimed to evaluate the correlation between the results of the evaluation protocol of the Functional Neurometry test and blood biomarkers indicative of anaemia, subclinical inflammation and/or endothelial dysfunction.

\section{Methodology}

A total of 412 male patients aged between 18 and 50years were evaluated by Functional Neurometry and Laboratory Testing. The subjects were divided into 2 groups: a) Control group (CONTROL, $\mathrm{n}=194)$ and b) Dysfunctional group (GD, $\mathrm{n}=218)$. The functional classification of the CONTROL group was established within the criteria of the expected standards of the Functional Neurometry method, such as: anxiety control/ functional reserve with an expected value of: $\geq 75 \%$; baroreflex index: $\geq 90 \%$; hemodynamic: $\leq 10 \%$; and physiological response between: $31.5^{\circ}$ and $31.5^{\circ}$.
All examinations with the Functional Neurometry method took place in an air-conditioned room at a temperature of $19^{\circ} \pm 2^{\circ} \mathrm{C}$. This assessment allows to indirectly investigate the functionality of the adrenal, liver, and the flexibility of blood vessel walls in the transport of functional oxygen, from the respiratory microcirculation (from the carotid sinus to the aortic arch) to the brain in 3 (three) positions: dorsal decubitus, standing up and orthostatic. Thus, the author called it DSO Analysis. The equipment and sensors of this method, already registered with the National Health Surveillance Agency (ANVISA), are periodically updated/calibrated, enabling the functional assessment of the autonomous nervous system (ANS), neurophysiological signals and brain mapping/neuroimaging, under the ANVISA Reg. No. 81403519002 .

ANS signals are measured by capturing low frequencies, separating them into sympathetic by means of vasoconstrictions, representing the recording of the effect of the perception of external stimuli by the frequencies 0.01 to $0.04 \mathrm{~Hz}$ and of the perception of internal stimuli by frequencies 0.04 to $0.20 \mathrm{~Hz}$, and also of the parasympathetic through vasodilation, expressed by frequencies 0.20 to $0.50 \mathrm{~Hz} .^{11}$

The specifications of each category of the DSO analysis organized by author Nelson Alves Pereira Júnior, mentioned in the second paragraph of the introduction to this study, involving the form of capture and analysis of biological signals by each sensor regulated in algorithms organized by the Fourier Transform, are recorded in the study by Ribas et al. ${ }^{2}$

All patients were evaluated at the Instituto do Cérebro de Pernambuco (ICerPE). Before evaluation by Functional Neurometry, the following measurements were taken: weight $[\mathrm{Kg}]$, height $[\mathrm{m}]$, waist circumference $[\mathrm{cm}]$ and body mass index. Patients usually seek ICerPE with the aim of undergoing complete assessments with Functional Neurometry and Quantitative Electroencephalography. Eligibility criteria: inclusion: patients who reported that they did not practice physical activity frequently, did not use supplements and did not have nutritional monitoring were included. Exclusion: patients who used glucose control, cholesterol and controlled medications were excluded.

After the evaluations with the Functional Neurometry of each patient, the physician of the team of this scientific research delivered to each patient a request for a blood test, already paid for by ICerPE, of the following biomarkers: red blood cells, haemoglobin, haematocrit, mean corpuscular volume-MCV; mean corpuscular haemoglobin-MCH; mean corpuscular haemoglobin con-centration$\mathrm{MCHC}$; red cell distribution width-RDW; total leukocytes-TL, ferritin, transferrin saturation-TS, B12, homocysteine, C-reactive protein-CRP; fibrinogen; 25-hydroxy-vitamin-D3; apolipoprotein A1Apo-A1; apolipoprotein B-Apo-B; glutamic oxalacetic transaminaseGOT; glutamic-pyruvic transaminase-GPT; gamma-glutamyl transpeptidase-Gamma GT; 8am cortisol; Immunoglobulin E-IgE; total cholesterol-TC; triglycerides-TG, and; high-density lipoproteinHDL and low-density lipoprotein-LDL.

The method of calculating the size of a sample for an infinite population was used:

$$
n=\frac{z^{2} * P^{*} Q}{e^{2}}
$$

$\mathbf{n}=$ sample size to be calculated;

$\mathbf{z}=1,96$ (according to the area under normal curve table for the given $95 \%$ confidence level). 
$\mathbf{P}=0,5=50 \%$ to succeed;

$\mathbf{Q}=0,5=50 \%$ of failure;

$\mathbf{e}=$ percentage estimate from true value;

$\mathrm{e}^{2}=(0,05)^{2}=5 \%$ (for an expected estimate of $5 \%$ of the true value).

\section{Chi-square test from the contingency table}

The categorical variables of the number of occurrences, that is, the frequencies, were converted into numerical variables and then analysed using the chi-square test with a significance level of $\mathrm{p} \leq 0.05^{*}$.

$$
\chi^{2}=\frac{N *\left(a * d-b^{*} c\right)^{2}}{(a+b) *(\mathrm{c}+\mathrm{d}) *(\mathrm{a}+\mathrm{c}) *(\mathrm{~b}+\mathrm{d})}
$$

\begin{tabular}{lll}
\hline & \multicolumn{2}{l}{ Biomarker out of expected value } \\
\hline & Yes & No \\
Dysfunctional Group, $\mathrm{n}=218$ & a & b \\
CONTROL Group, $\mathrm{n}=194$ & c & d
\end{tabular}

$\mathrm{a}=$ Dysfunctional with biomarker out of the expected value

$\mathrm{b}=$ Dysfunctional with biomarker within the expected value

$\mathrm{c}=\mathrm{CONTROL}$ group with biomarker out of the expected value

$\mathrm{d}=$ CONTROL group with biomarker within the expected value

$[\mathrm{a} ; \mathrm{d}]=$ Concordant variables;

$[\mathrm{b} ; \mathrm{c}]=$ Discordant variables

\section{Results}

In Table 1, the results of the CONTROL group, $\mathrm{n}=194$, were described as Median/(Minimum-Maximum) of each category assessed, as: Anxiety control/Functional reserve: 75.88/(72.0585.00) [Expected value $\geq 75 \%$ ]; Baroreflex index: $92.80 /(89.00-$ 95.98) [Expected value $\geq 90 \%$ ]; Hemodynamic: $10.00 /(09.00-10.00)$ [Expected value $\leq 10 \%$ ]; Physiological response: $32.25 /(29.00-33.73)$ [Reference value: 31.5 to 32.5 ] (Table 1).

The frequency of patients in the CONTROL group affected by their biomarkers is described in Table 2 below:

Table I Functional Neurometry exams of 194 male patients with functional results - Control group (CONT, $n=194)$

\begin{tabular}{|c|c|c|c|c|c|c|c|}
\hline \multicolumn{8}{|c|}{ Functional neurometry } \\
\hline AC & & BRI & & HD & & PR & \\
\hline Exp. Min & Med (Min-Max) & Exp. Min & Med (Min-Max) & Exp. Min & Med (Min-Max) & ER & Med (Min-Max) \\
\hline$\geq 75 \%$ & $75.88 /(72.05-85.00)$ & $\geq 90 \%$ & $92.80 /(89.00-95.98)$ & $\leq 10 \%$ & $10.00 /(09.00-10.00)$ & $31.5-32.5 \%$ & $32.25 /(29.00-33.73)$ \\
\hline
\end{tabular}

Control group, $(\mathrm{n}=194)$

Acronyms:AC, anxiety control; BRI, Baroreflex index; HD, aaodynamic; PR, physiological response; Exp, expected minimum Min; ER, expected range; - Med (MinMax, median/(Minimum-Maximum); descriptive statistics, data expressed in median/(Minimum-Maximum)

Table 2 Blood tests of 194 male patients of the functional group, Control group (CONT, n=194)

\begin{tabular}{|c|c|c|c|c|c|c|c|c|c|c|c|}
\hline \multicolumn{12}{|c|}{ Blood biomarkers } \\
\hline RBC & & & $\mathrm{Hb}$ & & & Hct & & & MCV & & \\
\hline $\begin{array}{l}\text { Reference } \\
\text { value }\end{array}$ & Results & $\%$ & $\begin{array}{l}\text { Reference } \\
\text { value }\end{array}$ & Results & $\%$ & $\begin{array}{l}\text { Reference } \\
\text { value }\end{array}$ & Results & $\%$ & $\begin{array}{l}\text { Reference } \\
\text { value }\end{array}$ & Results & $\%$ \\
\hline $\begin{array}{c}4.7-6.1 \\
\text { million/uL }\end{array}$ & $5 / 194$ & $2.58 \%$ & 14.0 a 18.0 & $13 / 194$ & $6.70 \%$ & $\begin{array}{l}42.0-52.0 \\
\%\end{array}$ & $5 / 194$ & $2.58 \%$ & $80.0-98.0$ & $14 / 194$ & $7.22 \%$ \\
\hline $\mathrm{MCH}$ & & & $\mathrm{MCHC}$ & & & $\begin{array}{l}\text { Total } \\
\text { leukocytes }\end{array}$ & & & RDW & & \\
\hline $\begin{array}{l}\text { Reference } \\
\text { value }\end{array}$ & Results & $\%$ & $\begin{array}{l}\text { Reference } \\
\text { value }\end{array}$ & Results & $\%$ & $\begin{array}{l}\text { Reference } \\
\text { value }\end{array}$ & Results & $\%$ & $\begin{array}{l}\text { Reference } \\
\text { value }\end{array}$ & Results & $\%$ \\
\hline $27-31$ & $8 / 194$ & $4.12 \%$ & $32-36$ & $14 / 194$ & $7.24 \%$ & $>6.000 / u L$ & $21 / 194$ & $10.82 \%$ & II - I5.5 & $2 / 194$ & I,03\% \\
\hline Serum ferritin & & & $\begin{array}{l}\text { Transferrin } \\
\text { saturation }\end{array}$ & & & $\mathrm{BI} 2$ vitamin & & & Homocysteine & & \\
\hline $\begin{array}{l}\text { Reference } \\
\text { value }\end{array}$ & Results & $\%$ & $\begin{array}{l}\text { Reference } \\
\text { value }\end{array}$ & Results & $\%$ & $\begin{array}{l}\text { Reference } \\
\text { value }\end{array}$ & Results & $\%$ & $\begin{array}{l}\text { Reference } \\
\text { value }\end{array}$ & Results & $\%$ \\
\hline $\begin{array}{l}30.0 \text { A } 400.0 \\
\mathrm{ng} / \mathrm{ml}\end{array}$ & $5 / 194$ & $2.58 \%$ & $20-50 \%$ & $8 / 194$ & $4.12 \%$ & $\begin{array}{l}210.0 \text { - } \\
980.0 \text { picog/ } \\
\mathrm{ml}\end{array}$ & $12 / 194$ & $6.19 \%$ & $\begin{array}{l}05-9.68 \\
\text { micromol/l }\end{array}$ & $4 / 194$ & $2.06 \%$ \\
\hline Cortisol & & & $\lg E$ & & & Eosinophils & & & Neutrophils & & \\
\hline $\begin{array}{l}\text { Reference } \\
\text { value }\end{array}$ & Results & $\%$ & $\begin{array}{l}\text { Reference } \\
\text { value }\end{array}$ & Results & $\%$ & $\begin{array}{l}\text { Reference } \\
\text { value }\end{array}$ & Results & $\%$ & $\begin{array}{l}\text { Reference } \\
\text { value }\end{array}$ & Results & $\%$ \\
\hline$<15$ & $21 / 194$ & $10.82 \%$ & $>200 \mathrm{UI} / \mathrm{ml}$ & $4 / 194$ & $2.06 \%$ & $>7$ & $13 / 194$ & $6.70 \%$ & $>55 \%$ & $9 / 194$ & $4.64 \%$ \\
\hline $\begin{array}{l}\text { C-reactive } \\
\text { protein }\end{array}$ & & & Fibrinogen & & & $\begin{array}{l}\text { 25-hydroxy- } \\
\text { vitamin D3 }\end{array}$ & & & APO-AI & & \\
\hline
\end{tabular}

Citation: Ribas RMG, Ribas VR, Oliveira DCL, et al.Association between functional neurometry test results and blood biomarkers indicative of anaemia, subclinical inflammation, and endothelial dysfunction. Int J Complement Alt Med. 202 I; I4(5):225-233. DOI: I0.15406/ijcam.202I.I4.00566 
Table Continued...

\section{Blood biomarkers}

\begin{tabular}{|c|c|c|c|c|c|c|c|c|c|c|c|}
\hline RBC & & & $\mathrm{Hb}$ & & & Hct & & & MCV & & \\
\hline $\begin{array}{l}\text { Reference } \\
\text { value }\end{array}$ & Results & $\%$ & $\begin{array}{l}\text { Reference } \\
\text { value }\end{array}$ & Results & $\%$ & $\begin{array}{l}\text { Reference } \\
\text { value }\end{array}$ & Results & $\%$ & $\begin{array}{l}\text { Reference } \\
\text { value }\end{array}$ & Results & $\%$ \\
\hline $\begin{array}{l}<0.3 \mathrm{mg} / \mathrm{dL} /< \\
5,0 \mathrm{mg} / \mathrm{l}\end{array}$ & $6 / 194$ & $3.09 \%$ & $\begin{array}{l}200-400 \\
\mathrm{mg} / \mathrm{dl}\end{array}$ & $4 / 194$ & $2.06 \%$ & $>40$ & $9 / 194$ & $4.64 \%$ & $\begin{array}{l}\mathrm{I} 04-202 \\
\mathrm{mg} / \mathrm{I} 00 \mathrm{ml}\end{array}$ & $13 / 194$ & $6.7 \%$ \\
\hline APO-B & & & GOT & & & GPT & & & GAMMA GT & & \\
\hline $\begin{array}{l}\text { Reference } \\
\text { value }\end{array}$ & Results & $\%$ & $\begin{array}{l}\text { Reference } \\
\text { value }\end{array}$ & Results & $\%$ & $\begin{array}{l}\text { Reference } \\
\text { value }\end{array}$ & Results & $\%$ & $\begin{array}{l}\text { Reference } \\
\text { value }\end{array}$ & Results & $\%$ \\
\hline $\begin{array}{l}66-133 \\
\mathrm{mg} / 100 \mathrm{ml}\end{array}$ & $5 / 194$ & $2.58 \%$ & $<34 \mathrm{U} / \mathrm{L}$ & $22 / 194$ & $11.34 \%$ & 10 - $49 \mathrm{U} / \mathrm{L}$ & 14/194 & $7.22 \%$ & $<38$ & $17 / 194$ & $8.76 \%$ \\
\hline $\begin{array}{l}\text { Total } \\
\text { Cholesterol }\end{array}$ & & & Triglycerides & & & HDL & & & LDL & & \\
\hline $\begin{array}{l}\text { Reference } \\
\text { value }\end{array}$ & Results & $\%$ & $\begin{array}{l}\text { Reference } \\
\text { value }\end{array}$ & Results & $\%$ & $\begin{array}{l}\text { Reference } \\
\text { value }\end{array}$ & Results & $\%$ & $\begin{array}{l}\text { Reference } \\
\text { value }\end{array}$ & Results & $\%$ \\
\hline$<190 \mathrm{mg} / \mathrm{dL}$ & $22 / 194$ & II.34\% & $<150 \mathrm{mg} / \mathrm{dL}$ & $21 / 194$ & $10.82 \%$ & $>40$ mg/dL & $10 / 194$ & $5.15 \%$ & $<130$ & $14 / 194$ & $7.22 \%$ \\
\hline $\mathrm{CT} / \mathrm{HDL}$ ratio & & & $\begin{array}{l}\text { TG/HDL } \\
\text { ratio }\end{array}$ & & & LDL/ HDL & & & $\begin{array}{l}\text { APO B/APO } \\
\text { AI ratio }\end{array}$ & & \\
\hline $\begin{array}{l}\text { Reference } \\
\text { value }\end{array}$ & Results & $\%$ & $\begin{array}{l}\text { Reference } \\
\text { value }\end{array}$ & Results & $\%$ & $\begin{array}{l}\text { Reference } \\
\text { value }\end{array}$ & Results & $\%$ & $\begin{array}{l}\text { Reference } \\
\text { value }\end{array}$ & Results & $\%$ \\
\hline$\leq 3.3$ & |4/| 94 & $7.22 \%$ & $\leq 2.5$ & $6 / 194$ & $3.09 \%$ & $\leq 2.3$ & $5 / 194$ & $2.58 \%$ & $\leq 0.69$ & $22 / 194$ & $11.34 \%$ \\
\hline
\end{tabular}

Control group, $(n=194)$

Acronyms: RBC, red blood cells; Hb, haemoglobin; Hct, haematocrit; MCV, mean corpuscular volume; $\mathrm{MCH}$, mean corpuscular haemoglobin; $\mathrm{MCHC}$, mean corpuscular haemoglobin concentration; RDW, red cell distribution width; TL, total leukocytes; TS, ferritin, transferrin saturation; HCY, homocysteine; CRP, C-reactive protein; Apo-AI, apolipoprotein AI;Apo-B, apolipoprotein B; GOT, glutamic oxalacetic transaminase; GPT, glutamic-pyruvic transaminase; TC, total cholesterol;TG, triglycerides; HDL, high-density lipoprotein; LDL, low-density lipoprotein

Categorical variables were converted into numerical variables and analysed by the chi-square test from the contingency table with a significance level of $p \leq 0.05 *$

In Table 3, the results allowed classifying the group as: Dysfunctional because they are incompatible with the minimum values expected in Functional Neurometry, $\mathrm{n}=218$, which were described as Median/(Minimum-Maximum) of each category assessed, as: Anxiety control/Functional reserve: 24.91/(10.11-69.17) [Minimum expected value of $75 \%$ ]; Baroreflex index: 86.54/(67.33-87.82) [Expected value
$>90 \%$ ]; Hemodynamic: 17.89/(15.74-24.41) [Expected value $\leq 10 \%$ ]; Physiological response: $24.98 /(22.60-28.60)$ [Reference value: 31.5 to 32.5 ] (Table 3).

The frequency of patients in the Dysfunctional group affected by their biomarkers is described in Table 4.

Table 3 Functional Neurometry exams of $2 \mid 8$ male patients with dysfunctional results, Dysfunctional group (DYSF, $n=2 \mid 8)$

\begin{tabular}{|c|c|c|c|c|c|c|c|}
\hline \multicolumn{8}{|c|}{ Functional neurometry } \\
\hline AC & & BRI & & HD & & $\mathbf{P R}$ & \\
\hline Exp. Min & Med (Min-Max) & Exp. Min & Med (Min-Max) & Exp. Min & Med (Min-Max) & ER & Med (Min-Max) \\
\hline$\geq 75 \%$ & $24.91 /(10.11-69.17)$ & $\geq 90 \%$ & $86.54 /(67.33-87.82)$ & $\leq 10 \%$ & $\mid 7.89 /(|5.74-24.4|)$ & $31.5-32.5 \%$ & $24.98 /(22.60-28.60)$ \\
\hline
\end{tabular}

Dysfunctional group, $(\mathrm{n}=2 \mid 8)$

Acronyms:AC, anxiety control; BRI, baroreflex index; HD, hemodynamic; PR, physiological response; Exp, expected minimum; ER, expected range; med (MinMax), median/(Minimum-Maximum); descriptive statistics, data expressed in median/(Minimum-Maximum)

Table 4 Blood tests of 218 male patients of the dysfunctional group, Dysfunctional group (DYSF, $n=218$ )

\begin{tabular}{|c|c|c|c|c|c|c|c|c|c|c|c|}
\hline \multicolumn{12}{|c|}{ Blood biomarkers } \\
\hline RBC & & & $\mathbf{H b}$ & & & Hct & & & MCV & & \\
\hline $\begin{array}{l}\text { Reference } \\
\text { value }\end{array}$ & Results & $\%$ & $\begin{array}{l}\text { Reference } \\
\text { value }\end{array}$ & Results & $\%$ & $\begin{array}{l}\text { Reference } \\
\text { value }\end{array}$ & Results & $\%$ & $\begin{array}{l}\text { Reference } \\
\text { value }\end{array}$ & Results & $\%$ \\
\hline $\begin{array}{c}4.7-6.1 \\
\text { million/uL }\end{array}$ & $2|/ 2| 8$ & $9.63 \%$ & $14.0-18.0$ & $94 / 218$ & $43.12 \%$ & $\begin{array}{l}42.0-52.0 \\
\%\end{array}$ & $52 / 218$ & $23.85 \%$ & $80.0-98.0$ & $114 / 218$ & $52.29 \%$ \\
\hline $\mathrm{MCH}$ & & & $\mathrm{MCHC}$ & & & $\begin{array}{l}\text { Total } \\
\text { leukocytes }\end{array}$ & & & RDW & & \\
\hline $\begin{array}{l}\text { Reference } \\
\text { value }\end{array}$ & Results & $\%$ & $\begin{array}{l}\text { Reference } \\
\text { value }\end{array}$ & Results & $\%$ & $\begin{array}{l}\text { Reference } \\
\text { value }\end{array}$ & Results & $\%$ & $\begin{array}{l}\text { Reference } \\
\text { value }\end{array}$ & Results & $\%$ \\
\hline
\end{tabular}

Citation: Ribas RMG, Ribas VR, Oliveira DCL, et al.Association between functional neurometry test results and blood biomarkers indicative of anaemia, subclinical inflammation, and endothelial dysfunction. Int J Complement Alt Med. 202 I; I4(5):225-233. DOI: I0.15406/ijcam.202I.I4.00566 
Table Continued...

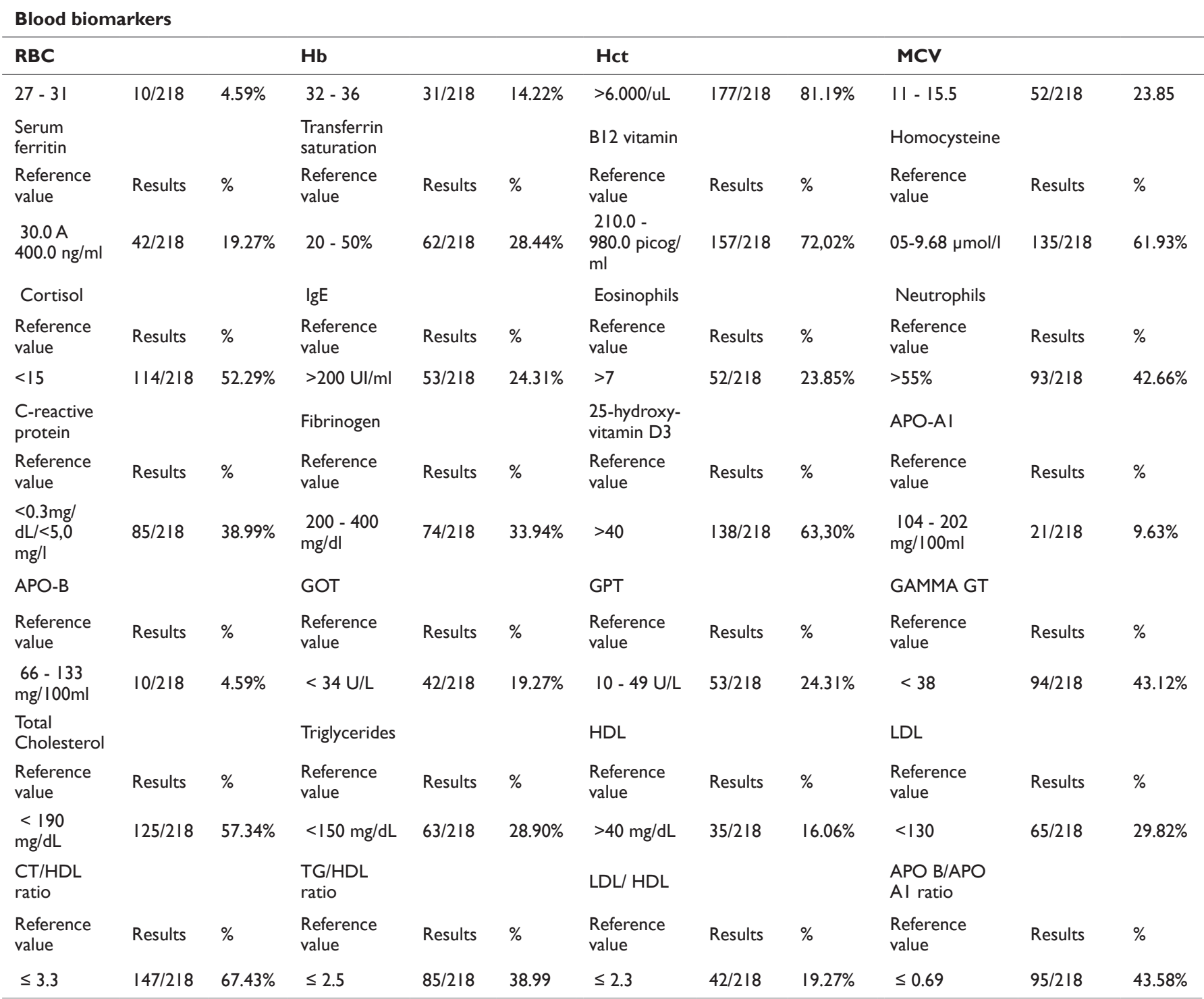

Dysfunctional group, $(\mathrm{n}=218)$

Acronyms: RBC, red blood cells; $\mathrm{Hb}$, haemoglobin; $\mathrm{Hct}$, haematocrit; $\mathrm{MCV}$, mean corpuscular volume; $\mathrm{MCH}$, mean corpuscular haemoglobin; $\mathrm{MCHC}$, mean corpuscular haemoglobin concentration; RDW, red cell distribution width; TL, total leukocytes; TS, ferritin, transferrin saturation; HCY, homocysteine; CRP, C-reactive protein; Apo-AI, apolipoprotein AI; Apo-B, apolipoprotein B; GOT, glutamic oxalacetic transaminase; GPT, glutamic-pyruvic transaminase; TC, total cholesterol;TG, triglycerides; HDL, high-density lipoprotein; LDL, low-density lipoprotein

Categorical variables were converted into numerical variables and analysed by the chi-square test from the contingency table with a significance level of $p \leq 0.05 *$

Finally, in Table 5 below, a significant association was observed the frequency of patients in the Dysfunctional group assessed by the between the frequency of affected patients in their biomarkers and Functional Neurometry method (N=218).

Table 5 Association between the Control Group (CONT, n=194) and the Dysfunctional Group (DYSF, n=218)

\begin{tabular}{|c|c|c|c|c|c|c|c|c|c|c|c|}
\hline \multicolumn{12}{|c|}{ Blood biomarkers } \\
\hline RBC & Results & & $\mathbf{H b}$ & Results & & Hct & Results & & MCV & Results & \\
\hline Groups & Yes/No & $\%$ & Groups & Yes/No & $\%$ & Groups & Yes/No & $\%$ & Groups & Yes/No & $\%$ \\
\hline $\begin{array}{l}\text { CONT, } N= \\
194\end{array}$ & $5 / 189$ & $2.58 \%$ & $\begin{array}{l}\text { CONT, N = } \\
194\end{array}$ & $13 / 18 \mid$ & $6.70 \%$ & $\begin{array}{l}\text { CONT, N = } \\
194\end{array}$ & $5 / 189$ & $2.58 \%$ & $\begin{array}{l}\text { CONT, N = } \\
194\end{array}$ & $14 / 180$ & $7.22 \%$ \\
\hline $\begin{array}{l}\text { DYSF, } N= \\
218\end{array}$ & $21 / 197$ & $9.63 \%$ & $\begin{array}{l}\text { DYSF, N = } \\
218\end{array}$ & $94 / 124$ & $43.12 \%$ & $\begin{array}{l}\text { DYSF, } N= \\
218\end{array}$ & $52 / 166$ & $23.85 \%$ & DYSF, $N=218$ & $1 / 4 / 104$ & $52.29 \%$ \\
\hline$P$ value & 0.0033 & & $\mathrm{P}$ value & $<0.0001$ & & $P$ value & $<0.0001$ & & $\mathrm{P}$ value & $<0.0001$ & \\
\hline $\mathrm{MCH}$ & Results & & $\mathrm{MCHC}$ & Results & & $\begin{array}{l}\text { Total } \\
\text { leukocytes }\end{array}$ & Results & & RDW & Results & \\
\hline
\end{tabular}

Citation: Ribas RMG, Ribas VR, Oliveira DCL, et al.Association between functional neurometry test results and blood biomarkers indicative of anaemia, subclinical inflammation, and endothelial dysfunction. Int J Complement Alt Med. 202 I; I4(5):225-233. DOI: I0.15406/ijcam.202I.I4.00566 
Table Continued..

\begin{tabular}{|c|c|c|c|c|c|c|c|c|c|c|c|}
\hline \multicolumn{12}{|c|}{ Blood biomarkers } \\
\hline RBC & Results & & $\mathbf{H b}$ & Results & & Hct & Results & & MCV & Results & \\
\hline Groups & Yes/No & $\%$ & Groups & Yes/No & $\%$ & Groups & Yes/No & $\%$ & Groups & Yes/No & $\%$ \\
\hline $\begin{array}{l}\text { CONT, N = } \\
194\end{array}$ & $8 / 186$ & $4.12 \%$ & $\begin{array}{l}\mathrm{CONT}, \mathrm{N}= \\
194\end{array}$ & $14 / 180$ & $7.24 \%$ & $\begin{array}{l}\text { CONT, N = } \\
194\end{array}$ & $21 / 173$ & $10.82 \%$ & $\begin{array}{l}\mathrm{CONT}, \mathrm{N}= \\
194\end{array}$ & $2 / 192$ & $1,03 \%$ \\
\hline $\begin{array}{l}\text { DYSF, } N= \\
218\end{array}$ & $10 / 208$ & $4.59 \%$ & $\begin{array}{l}\text { DYSF, } N= \\
218\end{array}$ & $31 / 187$ & $14.22 \%$ & $\begin{array}{l}\text { DYSF, } N= \\
218\end{array}$ & $|77 / 4|$ & $81.19 \%$ & DYSF, $N=218$ & $52 / 166$ & 23.85 \\
\hline$P$ value & 0.8183 & & $P$ value & 0.0229 & & $P$ value & $<0.0001$ & & $P$ value & $<0.0001$ & \\
\hline $\begin{array}{l}\text { Serum } \\
\text { ferritin }\end{array}$ & Results & & $\begin{array}{l}\text { Transferrin } \\
\text { saturation }\end{array}$ & Results & & $\mathrm{B} \mid 2$ vitamin & Results & & Homocysteine & Results & \\
\hline Groups & Yes/No & $\%$ & Groups & Yes/No & $\%$ & Groups & Yes/No & $\%$ & Groups & Yes/No & $\%$ \\
\hline $\begin{array}{l}\text { CONT, N = } \\
194\end{array}$ & $5 / 189$ & $2.58 \%$ & $\begin{array}{l}\mathrm{CONT}, \mathrm{N}= \\
194\end{array}$ & $8 / 186$ & $4.12 \%$ & $\begin{array}{l}\text { CONT, N = } \\
194\end{array}$ & $12 / 182$ & $6.19 \%$ & $\begin{array}{l}\mathrm{CONT}, \mathrm{N}= \\
194\end{array}$ & $4 / 190$ & $2.06 \%$ \\
\hline $\begin{array}{l}\text { DYSF, } N= \\
218\end{array}$ & $42 / 176$ & $19.27 \%$ & $\begin{array}{l}\text { DYSF, } N= \\
218\end{array}$ & $62 / 156$ & $28.44 \%$ & $\begin{array}{l}\text { DYSF, } N= \\
218\end{array}$ & $157 / 6 \mid$ & $72,02 \%$ & $\begin{array}{l}\text { DYSF, } N= \\
218\end{array}$ & $135 / 83$ & $61.93 \%$ \\
\hline$P$ value & $<0.0001$ & & $P$ value & $<0.0001$ & & $\mathrm{P}$ value & $<0.0001$ & & $\mathrm{P}$ value & $<0.0001$ & \\
\hline Cortisol & Results & & $\lg E$ & Results & & Eosinophils & Results & & Neutrophils & Results & \\
\hline Groups & Yes/No & $\%$ & Groups & Yes/No & $\%$ & Groups & Yes/No & $\%$ & Groups & Yes/No & $\%$ \\
\hline $\begin{array}{l}\text { CONT, N = } \\
194\end{array}$ & $21 / 173$ & $10.82 \%$ & $\begin{array}{l}\text { CONT, N = } \\
194\end{array}$ & $4 / 190$ & $2.06 \%$ & $\begin{array}{l}\text { CONT, N = } \\
194\end{array}$ & $|3 /| 8 \mid$ & $6.70 \%$ & $\begin{array}{l}\text { CONT, } N= \\
194\end{array}$ & $9 / 185$ & $4.64 \%$ \\
\hline $\begin{array}{l}\text { DYSF, } N= \\
218\end{array}$ & $1 / 4 / 104$ & $52.29 \%$ & $\begin{array}{l}\text { DYSF, } N= \\
218\end{array}$ & $53 / 165$ & $24.31 \%$ & $\begin{array}{l}\text { DYSF, } N= \\
218\end{array}$ & $52 / 166$ & $23.85 \%$ & $\begin{array}{l}\text { DYSF, } N= \\
218\end{array}$ & $93 / 125$ & $42.66 \%$ \\
\hline$P$ value & $<0.0001$ & & $P$ value & $<0.0001$ & & $P$ value & $<0.0001$ & & $\mathrm{P}$ value & $<0.0001$ & \\
\hline $\begin{array}{l}\text { C-reactive } \\
\text { protein }\end{array}$ & Results & & Fibrinogen & Results & & $\begin{array}{l}\text { 25-hydroxy- } \\
\text { vitamin D3 }\end{array}$ & Results & & APO-AI & Results & \\
\hline Groups & Yes/No & $\%$ & Groups & Yes/No & $\%$ & Groups & Yes/No & $\%$ & Groups & Yes/No & $\%$ \\
\hline $\begin{array}{l}\text { CONT, N = } \\
194\end{array}$ & $6 / 188$ & $3.09 \%$ & $\begin{array}{l}\text { CONT, N = } \\
194\end{array}$ & $4 / 190$ & $2.06 \%$ & $\begin{array}{l}\text { CONT, N = } \\
194\end{array}$ & $9 / 185$ & $4.64 \%$ & $\begin{array}{l}\text { CONT, } N= \\
194\end{array}$ & $|3 / 18|$ & $6.7 \%$ \\
\hline $\begin{array}{l}\text { DYSF, } N= \\
218\end{array}$ & $85 / 133$ & $38.99 \%$ & $\begin{array}{l}\text { DYSF, } N= \\
218\end{array}$ & $74 / / 44$ & $33.94 \%$ & $\begin{array}{l}\text { DYSF, } N= \\
218\end{array}$ & $138 / 80$ & $63,30 \%$ & $\begin{array}{l}\text { DYSF, } N= \\
218\end{array}$ & $21 / 197$ & $9.63 \%$ \\
\hline$P$ value & $<0.0001$ & & $P$ value & $<0.0001$ & & $P$ value & $<0.0001$ & & $P$ value & 0.2803 & \\
\hline APO-B & Results & & GOT & Results & & GPT & Results & & GAMMA GT & Results & \\
\hline Groups & Yes/No & $\%$ & Groups & Yes/No & $\%$ & Groups & Yes/No & $\%$ & Groups & Yes/No & $\%$ \\
\hline $\begin{array}{l}\text { CONT, N = } \\
194\end{array}$ & $5 / 189$ & $2.58 \%$ & $\begin{array}{l}\text { CONT, N = } \\
194\end{array}$ & $22 / 172$ & I I.34\% & $\begin{array}{l}\text { CONT, N = } \\
194\end{array}$ & $14 / 180$ & $7.22 \%$ & $\begin{array}{l}\text { CONT, } N= \\
194\end{array}$ & $17 / 177$ & $8.76 \%$ \\
\hline $\begin{array}{l}\text { DYSF, } N= \\
218\end{array}$ & $10 / 208$ & $4.59 \%$ & $\begin{array}{l}\text { DYSF, } N= \\
218\end{array}$ & $42 / 176$ & $19.27 \%$ & $\begin{array}{l}\text { DYSF, } N= \\
218\end{array}$ & $53 / 165$ & $24.31 \%$ & $\begin{array}{l}\text { DYSF, } N= \\
218\end{array}$ & $94 / 124$ & $43.12 \%$ \\
\hline$P$ value & 0.2770 & & $\mathrm{P}$ value & 0.0266 & & $\mathrm{P}$ value & $<0.0001$ & & $\mathrm{P}$ value & $<0.0001$ & \\
\hline $\begin{array}{l}\text { Total } \\
\text { Cholesterol }\end{array}$ & Results & & Triglycerides & Results & & HDL & Results & & LDL & Results & \\
\hline Groups & Yes/No & $\%$ & Groups & Yes/No & $\%$ & Groups & Yes/No & $\%$ & Groups & Yes/No & $\%$ \\
\hline $\begin{array}{l}\text { CONT, } N= \\
194\end{array}$ & $22 / 172$ & II.34\% & $\begin{array}{l}\text { CONT, N = } \\
194\end{array}$ & $21 / 173$ & $10.82 \%$ & $\begin{array}{l}\mathrm{CONT}, \mathrm{N}= \\
194\end{array}$ & $10 / 184$ & $5.15 \%$ & $\begin{array}{l}\text { CONT, N = } \\
194\end{array}$ & $14 / 180$ & $7.22 \%$ \\
\hline $\begin{array}{l}\text { DYSF, } N= \\
218\end{array}$ & $125 / 93$ & $57.34 \%$ & $\begin{array}{l}\text { DYSF, } N= \\
218\end{array}$ & $63 / 155$ & $28.90 \%$ & $\begin{array}{l}\text { DYSF, } N= \\
218\end{array}$ & $35 / 183$ & $16.06 \%$ & $\begin{array}{l}\text { DYSF, } N= \\
218\end{array}$ & $65 / 153$ & $29.82 \%$ \\
\hline$P$ value & $<0.0001$ & & $\mathrm{P}$ value & $<0.0001$ & & $P$ value & 0.0004 & & $\mathrm{P}$ value & $<0.0001$ & \\
\hline $\begin{array}{l}\mathrm{CT} / \mathrm{HDL} \\
\text { ratio }\end{array}$ & Results & & $\begin{array}{l}\text { TG/HDL } \\
\text { ratio }\end{array}$ & Results & & LDL/ HDL & Results & & $\begin{array}{l}\text { APO B/APO } \\
\text { Al ratio }\end{array}$ & Results & \\
\hline Groups & Yes/No & $\%$ & Groups & Yes/No & $\%$ & Groups & Yes/No & $\%$ & Groups & Yes/No & $\%$ \\
\hline
\end{tabular}


Table Continued...

\begin{tabular}{|c|c|c|c|c|c|c|c|c|c|c|c|}
\hline \multicolumn{12}{|c|}{ Blood biomarkers } \\
\hline RBC & Results & & $\mathbf{H b}$ & Results & & Hct & Results & & MCV & Results & \\
\hline $\begin{array}{l}\text { CONT, } N= \\
194\end{array}$ & $14 / 180$ & $7.22 \%$ & $\begin{array}{l}\text { CONT, } N= \\
194\end{array}$ & $6 / 188$ & $3.09 \%$ & $\begin{array}{l}\text { CONT, N = } \\
194\end{array}$ & $5 / 189$ & $2.58 \%$ & $\begin{array}{l}\mathrm{CONT}, \mathrm{N}= \\
194\end{array}$ & $22 / 172$ & $11.34 \%$ \\
\hline $\begin{array}{l}\text { DYSF, } N= \\
218\end{array}$ & |47/7 | & $67.43 \%$ & $\begin{array}{l}\text { DYSF, N = } \\
218\end{array}$ & $85 / 133$ & 38.99 & $\begin{array}{l}\text { DYSF, } N= \\
218\end{array}$ & $42 / 176$ & $19.27 \%$ & $\begin{array}{l}\text { DYSF, } N= \\
218\end{array}$ & $95 / 123$ & $43.58 \%$ \\
\hline
\end{tabular}

Total patients: 412

Control group, $(n=194)$

Dysfunctional group, $(\mathrm{n}=218)$

Acronyms: RBC, red blood cells; Hb, haemoglobin; Hct, haematocrit; MCV, mean corpuscular volume; $\mathrm{MCH}$, mean corpuscular haemoglobin; MCHC, mean corpuscular haemoglobin concentration; RDW, red cell distribution width; TL, total leukocytes; TS, ferritin, transferrin saturation; HCY, homocysteine; CRP, C-reactive protein; Apo-AI, apolipoprotein AI;Apo-B, apolipoprotein B; GOT, glutamic oxalacetic transaminase; GPT, glutamic-pyruvic transaminase; TC, total cholesterol;TG, triglycerides; HDL, high-density lipoprotein; LDL, low-density lipoprotein

Categorical variables were converted into numerical variables and analysed by the chi-square test from the contingency table with a significance level of $p \leq 0.05 *$

Thus, it was observed in table 5, higher frequencies of normocytic, microcytic, macrocytic or megaloblastic anaemia and anisocytosis in the dysfunctional group in their biomarkers, respectively: red cells [21 of 218, (9.63\%), p<0.0033]; haemoglobin [94 of 218, $(43.12 \%)$, $\mathrm{p}<0.0001]$; haematocrits [52 of $218,(23.85 \%), \mathrm{p}<0.0001$ ]; MCV [114 of $218,(52.29 \%), \mathrm{p}<0.0001$ ]; and RDW [52 out of $218,(23.85 \%)$, $\mathrm{p}<0.0001]$, when compared to the CONTROL group in their biomarkers: red blood cells [5 out of 194, $(2.58 \%)$ ]; haemoglobins [13 of 194, $(6.70 \%)]$; haematocrits [5 of 194, (2.58\%)]; MCV [14 of 194, (7.22\%)]; and RDW [2 of 194, (1.03\%)]. However, there was no significant association in cases of hypochromic anaemia, identified by the $\mathrm{MCH}$ and $\mathrm{MCHC}$ biomarkers in this study.

Higher frequencies of increased iron (close to the highest reference value of the laboratories) and/or low iron (close to the lowest reference value of the laboratories) in the bone marrow of the dysfunctional group through the serum ferritin biomarker: [42 of 218, (19.27\%), $\mathrm{p}<0.0001]$, when compared to the CONTROL group in their serum ferritin biomarkers [5 of 194, (2.58\%)].

Higher frequencies of patients with low vitamin B12: [157 out of $218,(72.02 \%), \mathrm{p}<0.0001]$ and high homocysteine: [135 out of 218 , $(61.93 \%), p<0.0001]$ from the dysfunctional group, when compared to the CONTROL group, vitamin B12: [12 out of 194, (6.19\%)] and Homocysteine: [4 out of 194, (2.06\%)], from the CONTROL group.

High frequency of patients with low cortisol (below $15 \mathrm{mcg} / \mathrm{dL}$ ) from 8:00 am [114 out of 218, (52.29\%), p<0.0001]; with high GPT values: [53 of $218,(24.31 \%), \mathrm{p}<0.0001$ ]; and Gamma GT: [94 out of $218,(43.12 \%), \mathrm{p}<0.0001]$ from the dysfunctional group, when compared to the $8: 00$ am cortisol [21 out of $194,(10.82 \%)]$, from the GPT [14 of 194, (7.22\%)] and Gamma GT [17 of 194, (8.76\%)] from the CONTROL group.

Higher frequency of patients with total leukocytes above 6000 and below 10,000 [177 out of $218,(81.19 \%), p<0.0001]$; high C-reactive protein (above $0.33 \mathrm{mg} / \mathrm{dL}$ ): [ 85 of $218,(38.99 \%), \mathrm{p}<0.0001$ ]; high fibrinogen (above $400 \mathrm{mg} / \mathrm{dL}$ ): [74 of 218, $(33.94 \%), p<0.0001]$ and low 25-hydroxy-vitamin-D3 (below $40 \mathrm{ng} / \mathrm{mL}$ ) [138 of $218,(63.30 \%$ ), $\mathrm{p}<0.0001]$ in the dysfunctional group, when compared to the total number of leukocytes above 6000 in the CONTROL group [21 out of 194, (10.82\%)]; C-reactive protein: [6 of 194, $(3.09 \%), \mathrm{p}<0.0001$ ]; fibrinogen: [4 of 194, (2.06\%)] and 25-hydroxy-vitamin-D3 [9 of 194, (4.64\%)] from the CONTROL group.
Higher frequency of patients with high TC (above $190 \mathrm{mg} / \mathrm{dL}$ ) [125 out of $218,(57.34 \%), \mathrm{p}<0.0001]$; high TG level (above $150 \mathrm{mg} /$ $\mathrm{dL}$ ): [63 of 218, $(28.90 \%), \mathrm{p}<0.0001]$; low HDL (Below $40 \mathrm{mg}$ ) $\mathrm{dL}$ ): [35 of 218, (16.06\%), $\mathrm{p}<0.0001]$; high LDL (Above $130 \mathrm{mg} /$ $\mathrm{dL}$ ): [65 of $218,(29.82 \%), \mathrm{p}<0.0001]$ and high TC/HDL (Above 3.3) [138/218, $(63,30 \%), \mathrm{p}<0.0001]$, high TG/HDL (Above 2.5) [138/218, $(63.30 \%), p<0.0001]$, high LDL/HDL (Above 2.3) [42 out of 218, (19.27\%), $<<0.0001]$ and high Apo B/Apo A1 ratios (Above 0.69) [95 out of $218,(63.30 \%), p<0.0001]$ of the dysfunctional group, when compared to the CONTROL group: TC [22 of 194, (11.34\%)]; TG: [21 of 194, (28.90\%)]; HDL: [10 of 194, (5.15\%)]; LDL: [14 of 194, $(7.22 \%)]$ and TC/HDL [14 of 194, (7.22\%)], TG/HDL [6 of 194, (3.09\%)], ratio LDL/HDL [5 of 194, $(2.58 \%)$ ] and Apo B/Apo A1 ratios [22 of $194,(11.34 \%)$ ], respectively.

\section{Discussion}

This study found an association between dysfunctional patterns of the autonomic nervous system measured by the Functional Neurometry method (low anxiety control, low baroreflex index, hemodynamic with slow blood flow velocity and altered physiological response) and conditions of anaemia, subclinical inflammation, and endothelial dysfunction.

The most frequent anaemias in this study were normocytic, microcytic, macrocytic, or megaloblastic and anisocytosis. The presence of anaemia demonstrates a strong indication of low functional oxygen in brain mitochondria. This low functional oxygen seems to reduce energy generation in the stages of the Krebs cycle and oxidative phosphorylation, making it difficult to achieve harmony between neuron firings. ${ }^{12}$

A randomized clinical trial with 577 non-breastfed children, 267 of them anaemic, ${ }^{13}$ demonstrated that iron supplements with micronutrients (Vitamin B2, B5, B6, biotin, ascorbic acid, lipoic acid, zinc and copper) were more effective in reducing the prevalence of anaemia.

The subclinical inflammation in this study was possible to be observed due to the results of patients with total leukocytes (above 6000 ), ${ }^{14}$ C-reactive protein (above $0.33 \mathrm{mg} / \mathrm{dL}$ ) ${ }^{15}$ fibrinogen (above $400 \mathrm{mg} / \mathrm{dL}$ ) ${ }^{16}$, low 25-hydroxy-vitamin-D3 (below $\left.40 \mathrm{ng} / \mathrm{ml}\right)^{17}$ and $\operatorname{IgE}$ (above $200 \mathrm{IU} / \mathrm{ml}) .^{18}$ 
Several pathological mechanisms that mediate inflammation cause proteolytic and oxidative damage to endothelial cells. ${ }^{14}$ In order to consider the criterion of subclinical inflammation, from the total number of leukocytes above 6000 , this study considered several findings ${ }^{19-23}$ which involved the correlation between coronary heart disease, acute myocardial infarction and total leukocyte count, where they demonstrated a consensus that values, on average, above $6000 \mathrm{~mm}^{3}$ of the total leukocytes, indicate subclinical inflammation.

The subclinical word associated with the term inflammation seems to have arisen in the context of the non-identification of diagnosis by health professionals who are still strictly linked to the reference values of the laboratories, and not to the recent indications of relevant studies.

While undiagnosed inflammations are called subclinical, it seems possible to also use the same term subclinical inflammation to separate from the term inflammation by pathogens (viruses, bacteria, fungi, and protozoa). However, when it is separated from the context of inflammation by pathogens, several authors justify the classification of subclinical inflammation by the presence of pollutants ${ }^{24}$ and foods that contain allergenic and inflammatory components. ${ }^{25}$

The total $\operatorname{IgE}$ biomarker can be indicated to investigate the occurrence of allergic reactions, in addition to being suitable also in suspected diseases caused by parasites ${ }^{26}$ or even bronchopulmonary aspergillosis, ${ }^{27}$ which is a fungal disease that affects the respiratory system.

When it comes to allergic reactions, some people attribute the increase in IgE to patients who are already affected by rhinitis, atopic dermatitis, or asthma. However, in the literature, there are findings that demonstrate the increase in $\operatorname{IgE}$ is due to induction by exposure to allergens, ${ }^{28}$ such as the consumption of bovine milk ${ }^{29}$ and gluten ${ }^{30}$ and this increase in $\operatorname{IgE}$ activates mast cells that release histamines, causing rhinitis, atopic dermatitis and asthma, ${ }^{18}$ and not the other way around.

Endothelial dysfunction in this study was identified by biomarkers CT (above 190mg/dL), TG (above 150mg/dL), HDL (Below 40mg/ $\mathrm{dL}$ ), LDL (Above $130 \mathrm{mg} / \mathrm{dL}$ ), ratio CT/HDL (Above 3.3), TG/HDL ratio (Above 2.5), LDL/HDL ratio (Above 2.3) and Apo B/Apo A1 ratio (Above 0.69$)^{31}$ of both groups and this analysis was based on one of the most relevant papers on the importance of HDL for the reduction of coronary risk published by Michael H. Davidson. ${ }^{32}$

Furthermore, data from large-scale Framingham studies seem to further reinforce this context, as they demonstrated a lower risk of coronary artery disease with higher HDL in the different LDL levels expressed in ratios..$^{33}$ This reasoning, which involves the various ratios of TC, TG and LDL with HDL, is based on the importance of HDL, which seems to be much more than a cholesterol transporter from the arteries and peripheral tissues to the liver.

According to Davidson (2011), HDL has antioxidant action, antiinflammatory action, has profibrinolytic action, antithrombotic action and even reverses cholesterol transport. ${ }^{32}$ However, an HDL below 40 can actually predispose people to a higher risk of coronary artery disease. $^{33}$

Regarding the Apo B/Apo A1 ratio, all Apo-B leaves the liver; whether large molecules (VLDL, IDL, LDL) or small molecules (LDL); all are atherogenic. However, all particles, which are transported back to the liver, that is, HDL, which contain Apo-A1, are antiatherogenic. ${ }^{34}$ There are about 40 proteins in HDL, with Apo A1 and Apo A2 corresponding to half the mass of HDL. HDL is a very proteinaceous and less lipidic particle. If it had a lot of lipid, it would decrease its density. ${ }^{35}$

But since each particle of molecules leaving the liver contains only one Apo-B, the total Apo-B in plasma directly reflects the number of potentially atherogenic lipoproteins circulating. It is Apo-B that causes LDL to become trapped in the arterial wall, that is, in cases with normal or even low LDL, but with a high Apo-B, it can indicate the presence of small and dense LDL, which are the most atherogenic, as they are rapidly oxidizable and promote an inflammatory response and plaque growth. ${ }^{34}$

Thus, based on the aforementioned evidence, the use of the relationships between Apo-B and Apo-A1 seems to offer an advantage as a cardiovascular risk biomarker, especially in normolipemic individuals. ${ }^{36}$

Another concern of this study in the context of endothelial dysfunction was related to homocysteine, since the dysfunctional group demonstrated by Functional Neurometry presented 135 of 218 individuals with homocysteine above 9.68. The study that guided the highest acceptable level of homocysteine of 9.68 for this work was the important manuscript by Chao et al., 1999, which evaluated in 192 patients the gradual effect of homocysteine on the severity and extent of atherosclerosis ${ }^{37}$ and of the 192 patients, 45 already had 1 obstructed respiratory microcirculation blood vessel. This allowed this dysfunctional feature eligibility above 9.68 in relation to homocysteine, which seems to serve the interests of clinic and research at the same time, considering that its early detection can reverse the risk of a coronary artery disease.

\section{Conclusion}

Finally, this study seems to have demonstrated the existence of a non-invasive tool, which is the Functional Neurometry method, which shows a strong association between the biomarkers responsible for identifying anaemia, subclinical inflammation and endothelial dysfunction, which can help in hospital screening sectors, directing the need to perform a blood test before medical care, in addition to its relevance already presented in other studies, as a precise instrument in respiratory manoeuvre training, such as: heart rate variability, respiratory amplitude, respiratory capacity and cardiac coherence.

\section{Acknowledgments}

None.

\section{Conflicts of interest}

Author declares there are no conflicts of interest.

\section{Funding}

None.

\section{References}

1. Ribas VR, Ribas RMG, Barros MGS, et al. Hemodynamics, baroreflex index and blood biomarkers of a patient who died after being affected by COVID-19: case report. Hematol Transfus Cell Ther. 2020;42(3):206-11.

2. Ribas VR, Guerra Ribas RdM, Viana MT, et al. The Functional Neurometry of Nelson Alves Pereira Júnior: An Advanced Method of Mapping and Biofeedback Training of the Autonomic Nervous System Functions. Journal of Psychology and Psychotherapy Research. 2020;7:1-19.

3. Choi JW, Thakur H, Cohen JR. Cardiac autonomic functioning across stress and reward: Links with depression in emerging adults. Int J Psychophysiol. 2021;168:1-8. 
4. Yang X, McCoy E, Anaya-Boig E, et al. The effects of traveling in different transport modes on galvanic skin response (GSR) as a measure of stress: An observational study. Environ Int. 2021;156:106764.

5. Urbancsek R, Forgacs IN, Papp TB, et al. Cardiovagal and sympathetic baroreflex regulation in heart failure. Orv Hetil. 2021;162(3):91-98.

6. Johannsen DL, Ravussin E. The role of mitochondria in health and disease. Curr Opin Pharmacol. 2009;9(6):780-786.

7. Hammoudi N, Ceccaldi A, Haymann JP, et al. Altered cardiac reserve is a determinant of exercise intolerance in sickle cell anemia patients. Eur $J$ Clin Invest. 2021:e13664.

8. Ionica M, Aburel OM, Vaduva A, et al. Vitamin D alleviates oxidative stress in adipose tissue and mesenteric vessels from obese patients with subclinical inflammation. Can J Physiol Pharmacol. 2020;98(2):85-92.

9. Hulsmans M, Van Dooren E, Holvoet P. Mitochondrial reactive oxygen species and risk of atherosclerosis. Curr Atheroscler Rep. 2012;14(3):264 276.

10. Alves Pereira NJ, Suliano LC, Lopes S. Resposta Neurométrica Computadorizada dos estímulos da Acupuntura auricular. Curitiba, PR: Faculdade IBRATE; 2016.

11. Alves Pereira NJ. Tratamento e prevenção de doenças crônicas não transmissíveis, baseado na variabilidade do sistema nervoso na neurometria. Revista Brasileira de Neurometria. 2017;1(1):27-46.

12. Ribas RMG, Oliveira DCL, Silva PC, et al. The different roles of Griffonia simplicifolia in the treatment of depression: a narrative review. Int J Complement Alt Med. 2021;14(3):167-172.

13. Rosado JL, González KE, del C Caamaño M, et al. Efficacy of different strategies to treat anemia in children: a randomized clinical trial. Nutrition Journal. 2010;9(1):1-10.

14. Madjid M, Awan I, Willerson JT, et al. Leukocyte count and coronary heart disease: implications for risk assessment. $\mathrm{J}$ Am Coll Cardiol. 2004;44(10):1945-1956.

15. Ridker PM, Hennekens CH, Buring JE, et al. C-reactive protein and other markers of inflammation in the prediction of cardiovascular disease in women. N Engl J Med. 2000;342(12):836-843.

16. Wildman RP, Muntner $\mathrm{P}$, Chen J, et al. Relation of inflammation to peripheral arterial disease in the national health and nutrition examination survey, 1999-2002. Am J Cardiol. 2005;96(11):1579-1583.

17. Melamed ML, Michos ED, Post W, et al. 25-hydroxyvitamin D levels and the risk of mortality in the general population. Arch Intern Med. 2008;168(15):1629-1637.

18. Thiara G, Goldman RD. Milk consumption and mucus production in children with asthma. Can Fam Physician. 2012;58(2):165-166.

19. Furman MI, Becker RC, Yarzebski J, et al. Effect of elevated leukocyte count on in-hospital mortality following acute myocardial infarction. Am J Cardiol. 1996;78(8):945-948.

20. Cannon CP, McCabe CH, Wilcox RG, et al. Association of white blood cell count with increased mortality in acute myocardial infarction and unstable angina pectoris. Am J Cardiol. 2001;87(5):636-639.
21. Barron HV, Harr SD, Radford MJ, et al. The association between white blood cell count and acute myocardial infarction mortality in patients $\geq 65$ years of age: findings from the cooperative cardiovascular project. Am J Cardiol. 2001;38(6):1654-1661.

22. Mueller C, Neumann F, Perruchoud A, et al. White blood cell count and long term mortality after non-ST elevation acute coronary syndrome treated with very early revascularisation. Heart. 2003;89(4):389-392.

23. Hung MJ, Cherng WJ. Comparison of white blood cell counts in acute myocardial infarction patients with significant versus insignificant coronary artery disease. Am J Cardiol. 2003;91(11):1339-1342.

24. Wolf K, Popp A, Schneider A, et al. Association between long-term exposure to air pollution and biomarkers related to insulin resistance, subclinical inflammation, and adipokines. Diabetes. 2016;65(11):33143326.

25. Lozinsky AC, Morais MBd. Eosinophilic colitis in infants. J Pediatr (Rio J). 2014;90:16-21

26. Rosario NA. IgE total em alergia respiratória e infecção por helmintos. $J$ Pediatr (Rio J). 2007;83(1):92-94

27. Carneiro ACC, Lemos ACM, Arruda SM, et al. Prevalência de aspergilose broncopulmonar alérgica em pacientes com fibrose cística na Bahia, Brasil. Jornal Brasileiro de Pneumologia. 2008;34(11):900-906.

28. Ibiapina CdC, Sarinho ESC, Camargos PAM, et al. Rinite alérgica: aspectos epidemiológicos, diagnósticos e terapêuticos. Jornal Brasileiro de Pneumologia. 2008;34:230-240.

29. Wandalsen GF, Cocco RR, Sole D. The different spectra of cow's milk allergy. J Pediatr (Rio J). 2003;79(6):561-566.

30. Czaja-Bulsa G. Non coeliac gluten sensitivity-A new disease with gluten intolerance. Clin Nutr. 2015;34(2):189-194.

31. Heitzer T, Schlinzig T, Krohn K, et al. Endothelial dysfunction, oxidative stress, and risk of cardiovascular events in patients with coronary artery disease. Circulation. 2001;104(22):2673-2678.

32. Davidson MH. Focusing on high-density lipoprotein for coronary heart disease risk reduction. Cardiol Clin. 2011;29(1):105-122.

33. Link JJ, Rohatgi A, de Lemos JA. HDL cholesterol: physiology, pathophysiology, and management. Curr Probl Cardiol. 2007;32(5):268314.v

34. Walldius G, Jungner I. The apoB/apoA-I ratio: a strong, new risk factor for cardiovascular disease and a target for lipid-lowering therapy-a review of the evidence. J Intern Med. 2006;259(5):493-519.

35. van der Steeg WA, Holme I, Boekholdt SM, et al. High-density lipoprotein cholesterol, high-density lipoprotein particle size, and apolipoprotein AI: significance for cardiovascular risk: the IDEAL and EPIC-Norfolk studies. Am J Cardiol. 2008;51(6):634-642.

36. Lima LM, Carvalho $\mathrm{MdG}$, et al. Indice apo $\mathrm{B} /$ apo $\mathrm{AI}$ e predição de risco cardiovascular. Arquivos Brasileiros de Cardiologia. 2007;88(6):e187-e190.

37. Chao CL, Tsai HH, Lee CM, et al. The graded effect of hyperhomocysteinemia on the severity and extent of coronary atherosclerosis. Atherosclerosis. 1999;147(2):379-386. 